\title{
Effects of fear of falling and activity restriction on normal and dual task walking in community dwelling older adults
}

\author{
Orna A. Donoghue*, Hilary Cronin, George M. Savva, Claire O’Regan, Rose Anne Kenny \\ The Irish Longitudinal Study on Ageing (TILDA), Trinity College Dublin, Ireland
}

\section{A R T I C L E I N F O}

\section{Article history:}

Received 25 April 2012

Received in revised form 12 September 2012

Accepted 30 October 2012

\section{Keywords:}

Gait

Anxiety

Falls

Ageing

Balance

\begin{abstract}
A B S T R A C T
Fear of falling (FOF) is associated with poor physical and psychosocial health and can have debilitating consequences especially when it leads to activity restriction. This study examined whether normal and dual task gait disruptions were independently associated with FOF and activity restriction or if they were fully explained by impaired health status. Data was obtained from The Irish Longitudinal Study on Ageing (TILDA). Community dwelling adults $\geq 65$ years, with a Mini-Mental State Examination score $\geq 18$ and who completed a gait assessment $(n=1307)$ were divided into three groups: no FOF, FOF but no activity restriction (FOF-NAR), FOF with activity restriction (FOF-AR). Physical, psychosocial and cognitive measures were obtained and gait characteristics were assessed using a GAITRite ${ }^{\mathbb{R}}$ mat during normal and dual task (cognitive) walking. After adjusting for sociodemographics, physical, mental and cognitive health, FOF was associated with reduced gait speed and stride length and increased double support phase and step width in normal and dual task conditions; these changes were most pronounced in those who restrict activities as a result of FOF. These gait changes may be associated with an increased fall risk, however some changes especially increased step width may also reflect positive, compensatory adaptations to FOF. The results also highlight the importance of treating underlying health impairments and preventing the transition from FOF to activity restriction.
\end{abstract}

(c) 2012 Elsevier B.V. All rights reserved.

\section{Introduction}

Fear of falling (FOF) is common in older adults, particularly females and is associated with a history of falls, gait and balance disorders, depression, poor self-rated health and reduced social activity [1-5]. It can affect an individual in two ways. The first is increased awareness leading to more cautious behaviour and positive fall prevention strategies. The second is avoidance of activities which can lead to social isolation, physical deconditioning, reduced functional capacity and frailty which can increase the risk of falls and exacerbate FOF [1,5-7].

Despite the strong association between falls and gait, limited research has examined independent associations between FOF and gait. Existing studies have found reduced gait speed [8-10], reduced step or stride length $[9,11]$, increased step width and increased double support phase (DSP) [9] in those with FOF but similar changes are observed with increasing age [12,13], depression [14] and with a history of falls [15], all of which are also associated with FOF. Rochat et al. [16] found similar gait

\footnotetext{
* Corresponding author at: The Irish Longitudinal Study on Ageing (TILDA), Lincoln Gate, Trinity College Dublin, Dublin 2, Ireland. Tel.: +353 1896 4391; fax: +35318962451.

E-mail address: odonogh@tcd.ie (O.A. Donoghue).
}

changes between groups with no FOF, FOF alone and FOF with activity restriction but while the differences between the no FOF and FOF alone groups were fully explained by confounding variables, those with FOF and activity restriction had decreased gait performance and increased gait variability independent of health and functional status.

Gage et al. [17] suggested that anxiety associated with FOF may reduce the cognitive resources available for gait and balance control which may lead to gait disruptions. As dual task walking requires greater attention than normal walking, greater disruptions would be expected in dual task conditions. To date, only one study has examined dual task gait in older adults with and without FOF [10]. Reelick et al. [10] reported reduced velocity which explained increased gait variability among those with FOF but found no effect of FOF on trunk sway or cognitive task performance.

Gait disturbances are modifiable risk factors for falls, functional decline and other adverse health outcomes [18] and so it is important to understand how FOF affects gait. Adjusting for known confounders such as socio-demographics, depression, co-morbidity and cognitive function will also help determine if gait changes associated with FOF can be attributed to differences in physical, mental or cognitive health. Therefore, there were two aims in this study: (i) to examine the relationship between FOF, activity 
restriction and gait characteristics in normal and dual task walking and (ii) to determine if these relationships persist after adjusting for potentially underlying factors.

\section{Methods \\ 2.1. Study design}

The Irish Longitudinal Study on Ageing (TILDA) is a large prospective cohort study examining the social, economic and health circumstances of community dwelling older adults in Ireland. This study is based on the first wave of data collected from October 2009 to July 2011. Details of the study design and data collection are available elsewhere [19]. Briefly, the sampling frame is the Irish Geodirectory, a listing of all residential addresses in the Republic of Ireland. A clustered sample of addresses was chosen and all household residents aged 50 or over and their spouses/partners (of any age) were eligible to participate. Ethical approval was obtained from Trinity College Dublin Research Ethics Committee and written informed consent was obtained from 8504 participants.

\subsection{Data collection}

There were three parts to data collection: (i) a computer-assisted personal interview that included detailed questions on socio-demographics, wealth, health, lifestyle, social support and participation, use of health and social care and attitudes to ageing; (ii) a self-administered questionnaire; and (iii) a health assessment carried out by research nurses. Inclusion criteria for this study were age $\geq 65$ years, no history of Parkinson's disease, Alzheimer's disease or dementia, Mini-Mental State Examination (MMSE) score of $\geq 18$ and ability to complete the gait assessment without the use of physical aids and with a minimum of eight steps. Data was available on 1307 participants following these exclusions.

Sociodemographic variables such as age, sex and education (primary, secondary, tertiary) were obtained. The number of doctor diagnosed chronic conditions was obtained from the following list: heart attack or heart failure or angina, cataracts, stroke, diabetes, lung disease, asthma, arthritis, osteoporosis, cancer, peptic ulcer, hip fracture, hypertension and high cholesterol. Grip strength was assessed using a Baseline $^{\mathbb{B}}$ hydraulic hand dynamometer. Two trials were averaged on each hand and the highest value was used in the analysis. Depression was assessed using the 20-item Centre for Epidemiological Studies Depression (CES-D) scale.

Fear of falling was assessed with the question "Are you afraid of falling?". Those that answered 'Yes' were asked "Do you ever limit your activities, for example, what you do or where you go, because you are afraid of falling?". This allowed participants to be classified into three groups - no FOF, FOF but no activity restrictions (FOF-NAR) and FOF with activity restrictions (FOF-AR). It is important to note that this activity restriction question addresses both physical and social activity limitations. The number of falls in the previous year was recorded and categorised into 0,1 or $2+$ falls.

Global cognition was assessed with the MMSE while executive function and visual scanning were assessed using the Colour Trails Test [20]. In Colour Trail 1, participants drew a line connecting circles numbered 1-25 in consecutive order. In Colour Trail 2, participants connected these numbered circles but alternated between pink and yellow circles, i.e. pink 1, yellow 2, pink 3 etc. Each test was timed and the difference (trail 1 - trail 2) was used as a measure of executive function. A choice reaction time (CRT) test used a computer based program to assess concentration and speed of processing. Participants depressed a button on the keyboard to initiate the test and maintained this position while they waited for a Yes/No stimulus to appear on screen. When the stimulus appeared, the participant released the button and pressed another corresponding Yes/No button on the keyboard. The time from appearance of the stimulus to pressing this button was the total reaction time.

Gait assessment took place using a $4.88 \mathrm{~m}$ computerised walkway with embedded pressure sensors (GAITRite ${ }^{\mathbb{R}}$, CIR Systems Inc., New York, USA). Participants performed two walks at their normal pace followed by two walks under cognitive dual task conditions. The cognitive task was to recite alternate letters of the alphabet (A-C-E, etc.). The number of letters attempted and the number correct were recorded. Participants started and finished $2.5 \mathrm{~m}$ before and $2 \mathrm{~m}$ after the walkway to allow for acceleration and deceleration. Mean gait speed, stride length, step width and DSP were obtained along with standard deviations (SDs) of stride length and stride time. As the SDs were related to the mean values for each variable [21], coefficient of variation $(\mathrm{CV})$ was calculated $(\mathrm{SD} / \mathrm{mean} \times 100)$ and used to indicate stride-to-stride variability in stride length and stride time. These gait variables were chosen for analysis because of their associations with adverse outcomes such as falls in older adults [22]. While step parameters may vary between right and left sides, stride parameters include data for the left step followed by the right step (or vice versa). Therefore, there is little variation between right and left stride-based data so we combined data for the right and left sides for analysis. Based on a minimum of eight steps (range 8-22 steps for normal walk; 826 steps for cognitive walk), this provided a minimum of six strides for analysis.

\subsection{Statistical analysis}

Statistical analysis was conducted using Stata v12 (StataCorp LP, TX, USA). All analyses were weighted with respect to age, sex and education to the Quarterly
National Household Survey (2010) to ensure that data were nationally representative. Data were further weighted by health status and sociodemographic factors to account for those who did not attend a health assessment. Baseline characteristics of each group were compared using regression analysis for continuous variables and chi-squared tests for categorical variables. Mean gait variables were normally distributed and regression analysis was used to examine differences between groups using the no FOF group as the reference. Gait variability measures were positively skewed so quantile regression was used to estimate the differences in the medians and minimise the influence of high values. An additional advantage of this approach over transformations is that it keeps the coefficients in their original units allowing a more intuitive interpretation. For each gait parameter, two models were estimated. Model 1 adjusted for age, sex, height and education while Model 2 additionally adjusted for chronic conditions, number of medications, grip strength, CES-D score, total reaction time, Colour Trail time difference and number of falls in the previous year. Complete covariate data was available for 1207 (92.3\%) and 1196 (91.5\%) participants for the normal and dual task walk respectively. Complete case analysis was used for multivariate analyses, i.e. all missing data were treated as missing at random. Significance level was set at $p<0.05$.

\section{Results}

In this population based sample (mean age 71.3 years; range 65-93 years), $73.5 \%$ of participants $(n=961)$ reported no FOF, $19.1 \%$ reported FOF but no activity restriction $(n=250)$ while $7.5 \%$ reported FOF with activity restriction $(n=96)$. Baseline characteristics for each group are provided in Table 1. Both FOF groups were older, more likely to be female and a faller and had poorer physical and mental health than the no FOF group $(p<0.05)$. There was no difference between groups in global cognition (MMSE) but the FOF-AR group had a longer choice reaction time and Colour Trail time difference than the no FOF group $(p<0.05)$.

Table 2 outlines the gait characteristics for the normal and dual task walk for each group. In the normal walk, a progressive deterioration in gait characteristics was observed moving from the no FOF to FOF-NAR and FOF-AR groups. Specifically, both FOF groups demonstrated reduced gait speed and stride length and increased DSP and stride time variability $(p<0.05)$. Similar univariate differences in gait speed, stride length and DSP were observed in the dual task walk $(p<0.05)$ while gait variability was non-significantly higher in both FOF groups. In the cognitive task, both groups attempted and recited correctly more letters than the no FOF group, but there was no difference between groups in the percentage of letters recited correctly.

Table 3 provides the coefficients from the multivariate regression analysis illustrating the magnitude of differences between groups using the no FOF group as the reference. After adjusting for age, sex, height and education, both FOF groups walked more slowly, took shorter steps and had increased step width and DSP compared to the no FOF group in both normal and dual task walking conditions $(p<0.05)$. Additionally adjusting for chronic conditions, number of medications, grip strength, CES-D score, total reaction time, Colour Trail time difference and number of falls reduced the coefficients for all variables but the differences observed in Model 1 remained statistically significant for almost all mean gait variables. The reduction in gait speed for the FOF-AR group $(p=0.051)$ and stride length for the FOF-NAR group $(p=0.057)$ in dual task conditions were also very close to the significance level chosen.

In summary, FOF with and without activity restriction was independently associated with slower gait speed, shorter stride length and increased step width and DSP both in normal and dual task walking. Effects on DSP and step width were similar in both groups however, reductions in gait speed and stride length were more pronounced in the FOF-AR group. There was no difference in stride length variability or stride time variability between groups in either normal or dual task walking $(p>0.05)$. 
Table 1

Baseline characteristics for no FOF, FOF-NAR and FOF-AR groups.

\begin{tabular}{|c|c|c|c|}
\hline & No FOF $(n=961)$ & FOF-NAR $(n=250)$ & FOF-AR $(n=96)$ \\
\hline Age (years) & $72.3 \pm 5.6$ & $74.9 \pm 5.8^{* * *}$ & $73.9 \pm 5.6$ \\
\hline Height (m) & $1.64 \pm 0.09$ & $1.61 \pm 0.08^{* * *}$ & $1.59 \pm 0.09^{* * *}$ \\
\hline Sex (female) & 46 & $78^{* * *}$ & $85^{* * *}$ \\
\hline \multicolumn{4}{|l|}{ Education } \\
\hline Primary & 55 & 58 & 60 \\
\hline Secondary & 32 & 30 & 30 \\
\hline Tertiary & 14 & 11 & 10 \\
\hline Chronic conditions & $2.1 \pm 1.5$ & $2.9 \pm 1.8^{* * *}$ & $3.2 \pm 1.6^{* * *}$ \\
\hline Medications & $1.7 \pm 1.1$ & $2.1 \pm 1.0^{* * *}$ & $2.2 \pm 0.9^{* * *}$ \\
\hline Maximum grip strength (kg) & $25.6 \pm 8.8$ & $19.6 \pm 6.9^{* * *}$ & $19.0 \pm 6.8^{* * *}$ \\
\hline \multicolumn{4}{|l|}{ Fall in previous year } \\
\hline 0 falls & 82 & 70 & 63 \\
\hline 1 fall & 14 & $18^{* * *}$ & $24^{* * *}$ \\
\hline $2+$ falls & 4 & 12 & 14 \\
\hline CES-D score & $4.7 \pm 5.6$ & $6.8 \pm 7.3^{* *}$ & $10.6 \pm 10.7^{* *, \ddagger}$ \\
\hline MMSE & $27.7 \pm 2.2$ & $28.0 \pm 2.1$ & $27.5 \pm 2.0$ \\
\hline Choice reaction time (ms) & $918 \pm 318$ & $978 \pm 406$ & $1017 \pm 332^{*}$ \\
\hline Colour Trail difference (s) & $64.2 \pm 32.3$ & $69.5 \pm 33.6$ & $75.8 \pm 27.4^{* *}$ \\
\hline
\end{tabular}

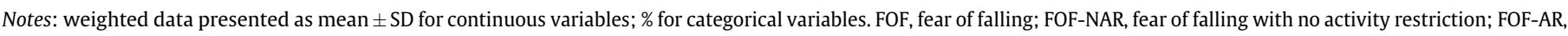
fear of falling with activity restriction; CES-D, Centre for Epidemiological Studies Depression scale; MMSE, Mini-Mental State Examination.

$p<0.05$ : FOF-NAR and FOF-AR groups significantly different to no FOF group.

${ }^{* *} p<0.01$ : FOF-NAR and FOF-AR groups significantly different to no FOF group.

$p<0.001$ : FOF-NAR and FOF-AR groups significantly different to no FOF group.

$\ddagger p<0.05$ : FOF-NAR significantly different to FOF-AR group.

Table 2

Gait characteristics of each group during the normal and dual task walk.

\begin{tabular}{|c|c|c|c|}
\hline & $\begin{array}{l}\text { No FOF } \\
\text { mean } \pm S D\end{array}$ & $\begin{array}{l}\text { FOF-NAR } \\
\text { mean } \pm \text { SD }\end{array}$ & $\begin{array}{l}\text { FOF-AR } \\
\text { mean } \pm \text { SD }\end{array}$ \\
\hline \multicolumn{4}{|l|}{ Normal walk } \\
\hline Gait speed $(\mathrm{m} / \mathrm{s})$ & $1.28 \pm 0.19$ & $1.20 \pm 0.22^{* * *}$ & $1.13 \pm 0.25^{* * *}$ ঐ\# \\
\hline Stride length (m) & $1.36 \pm 0.17$ & $1.26 \pm 0.18^{* * *}$ & $1.19 \pm 0.20^{* * *}$, \\
\hline Step width (cm) & $8.69 \pm 2.91$ & $8.74 \pm 3.04$ & $9.05 \pm 3.17$ \\
\hline DSP $(\%)$ & $26.53 \pm 4.24$ & $28.28 \pm 4.70^{* * *}$ & $29.14 \pm 5.71^{* * *}$ \\
\hline Stride length CV (\%) & $2.05(1.47-2.80)$ & $2.33(1.65-3.02)^{* *}$ & $2.15(1.61-3.22)$ \\
\hline Stride time CV $(\%)$ & $1.76(1.32-2.34)$ & $1.94(1.39-2.48)^{* *}$ & $2.03(1.69-2.69)^{* *}$ \\
\hline \multicolumn{4}{|c|}{ Dual task (cognitive) walk } \\
\hline Gait speed $(\mathrm{m} / \mathrm{s})$ & $1.04 \pm 0.24$ & $0.96 \pm 0.27^{* * *}$ & $0.93 \pm 0.26^{* * *}$ \\
\hline Stride length (m) & $1.28 \pm 0.21$ & $1.17 \pm 0.22^{* * *}$ & $1.12 \pm 0.21^{* * *}$ \\
\hline Step width $(\mathrm{cm})$ & $9.12 \pm 3.52$ & $9.26 \pm 3.62$ & $9.53 \pm 3.48$ \\
\hline DSP (\%) & $28.18 \pm 5.16$ & $30.25 \pm 6.08^{* * * *}$ & $30.99 \pm 5.67^{* * * *}$ \\
\hline Stride length CV (\%) & $3.12(2.22-4.48)$ & $3.45(2.42-4.94)^{*}$ & $3.42(2.16-5.22)$ \\
\hline Stride time CV (\%) & $3.35(2.25-5.49)$ & $3.68(2.55-5.98)$ & $3.68(2.51-5.40)$ \\
\hline Letters attempted & $7.6 \pm 2.1$ & $8.3 \pm 2.3^{* * *}$ & $8.3 \pm 2.3^{* *}$ \\
\hline Letters correct & $6.1 \pm 2.2$ & $6.6 \pm 2.4^{* *}$ & $6.6 \pm 2.5$ \\
\hline Letters correct (\%) & 80.7 & 80.3 & 79.0 \\
\hline
\end{tabular}

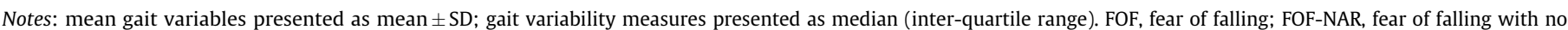
activity restriction; FOF-AR, fear of falling with activity restriction; DSP, double support phase; CV, coefficient of variation.

${ }^{*} p<0.05$ : FOF-NAR and FOF-AR groups significantly different to no FOF group.

** $p<0.01$ : FOF-NAR and FOF-AR groups significantly different to no FOF group.

$p<0.001$ : FOF-NAR and FOF-AR groups significantly different to no FOF group.

$* p<0.05$ : FOF-NAR significantly different to FOF-AR group.

\# $p<0.01$ : FOF-NAR significantly different to FOF-AR group.

\section{Discussion}

In this nationally representative, older Irish population, the prevalence of FOF with no activity restriction was $19 \%$ while $7.6 \%$ reported FOF with activity restriction. This is within the ranges of $11.2-39.9 \%$ and $5.2-43.4 \%$ respectively reported previously for community dwelling older adults $[4,5,16,23,24]$. Our results show that FOF and activity restriction are associated with deterioration in gait performance during normal and dual task walking independent of physical and mental health and cognitive function. These reductions in gait speed and stride length and increases in DSP and step width reflect a more cautious walking pattern and tended to be more pronounced in the FOF-AR group suggesting a gradual deterioration as FOF becomes more severe. However, FOF had no independent effect on gait variability suggesting that FOF does not cause gait to become more unstable.

There are conflicting opinions about the impact of these changes in gait characteristics. A slower than preferred gait speed and reduced step length have been associated with increased head and pelvic accelerations $[25,26]$ which may impair head control and gaze stabilisation, inadvertently leading to less stable gait [27]. Reduced gait speed and step length, increased DSP, stride/step length variability and swing time variability have also been shown to independently predict falls in older adults $[22,28]$ and therefore independent effects on some of these variables may place both FOF groups at increased risk of falls. 
Table 3

Regression analysis examining gait variables and FOF groups.

\begin{tabular}{|c|c|c|c|c|c|}
\hline & \multirow[t]{3}{*}{ No FOF } & \multicolumn{2}{|l|}{ Model 1} & \multicolumn{2}{|l|}{ Model 2} \\
\hline & & FOF-NAR & FOF-AR & FOF-NAR & FOF-AR \\
\hline & & $\beta$ coeff. & $\beta$ coeff. & $\beta$ coeff. & $\beta$ coeff. \\
\hline \multicolumn{6}{|l|}{ Normal walk } \\
\hline Gait speed (m/s) & Reference & $-5.87^{* * *}$ & $-15.12^{* * *}$ & $-4.18^{* *}$ & $-7.30^{* * *}$ \\
\hline Stride length (m) & Reference & $-4.67^{* * *}$ & $-11.20^{* * *}$ & $-3.41^{* *}$ & $-5.69^{* * *}$ \\
\hline Step width (cm) & Reference & $0.59^{* *}$ & $0.87^{*}$ & $0.55^{* *}$ & $0.71^{*}$ \\
\hline DSP $(\%)$ & Reference & $1.35^{* * *}$ & $3.21^{* * *}$ & $1.10^{* *}$ & $1.15^{*}$ \\
\hline Stride length CV (\%) & Reference & 0.13 & 0.19 & 0.07 & 0.15 \\
\hline Stride time CV (\%) & Reference & 0.08 & 0.17 & 0.03 & 0.17 \\
\hline \multicolumn{6}{|c|}{ Dual task (cognitive) walk } \\
\hline Gait speed (m/s) & Reference & $-4.82^{* *}$ & $-8.15^{* *}$ & $-3.50^{*}$ & -5.20 \\
\hline Stride length (m) & Reference & $-4.27^{* * *}$ & $-8.78^{* * *}$ & -2.38 & $-5.67^{* *}$ \\
\hline Step width $(\mathrm{cm})$ & Reference & $0.68^{* *}$ & $1.12^{* *}$ & $0.65^{*}$ & $0.82^{*}$ \\
\hline DSP (\%) & Reference & $1.27^{* *}$ & $2.11^{* * *}$ & $0.98^{*}$ & $1.44^{*}$ \\
\hline Stride length CV (\%) & Reference & 0.16 & 0.26 & 0.00 & 0.24 \\
\hline Stride time CV (\%) & Reference & 0.39 & 0.39 & 0.41 & 0.71 \\
\hline
\end{tabular}

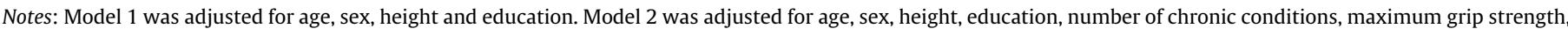
depressive symptoms (CES-D score), number of medications, total reaction time, Colour Trail time difference and number of falls in the past year.

${ }^{*} p<0.05$

*** $p<0.01$

$p<0.001$

Conversely, other studies suggest that reduced gait speed and step length in older adults may be a compensatory strategy to maintain head and pelvic stability. However, while individuals with low physiological fall risk have been shown to use this strategy to maintain stability, those with high physiological fall risk do not $[12,27]$ suggesting that the optimal and beneficial level of gait adaptation may also depend on physiological status. In this study, the more pronounced changes in gait speed and stride length were observed in the FOF-AR group who also had poorer health status and avoided activities such as walking. These gait changes may have been part of a compensatory strategy and while they did not coincide with an increase in stride-to-stride variability, it is possible that they may have been less effective in maintaining stability compared to the FOF-NAR group. However measurement of simultaneous head and pelvic accelerations or prospective analysis of falls would be required to assess this. Alternatively, reduced activity and the consequent decline in lower limb strength may explain the more pronounced reductions in gait speed and stride length.

Our findings also contribute to the limited research on FOF and dual task gait. Dual tasking is more reflective of everyday challenges and therefore ability to complete an independent cognitive task while walking may be particularly relevant for adults with FOF. Both FOF groups walked more slowly and therefore attempted and recited correctly more letters than the no FOF group but there was no difference in the \% of letters correct. Gait performance declined in dual task conditions in all groups however, dual cost or relative change from normal to dual task conditions was the same in all groups (data not shown) indicating that the FOF groups did not require additional cognitive input for the task. In addition, FOF was not independently associated with a greater level of gait instability. It is possible that the dual task used in our study provided cueing for participants to match their steps to which distracted them from anxiety associated with walking. Other studies have found that healthy adults walk more slowly, with reduced cadence, shorter steps and greater double support in anxiety inducing conditions (walking on an elevated walkway) which helped to improve dynamic stability and reduce fall risk [29]. Increased step width is another commonly used strategy to improve stability and was observed in both FOF groups. Therefore, it can be argued that the gait adaptations independently associated with FOF may be, at least partly attributed to stabilising strategies.
The main strength of this analysis is that it is the largest population based, nationally representative study to examine FOF and detailed spatiotemporal gait parameters to date. The extensive background information also allowed the data to be adjusted for multiple confounding factors. The main limitation is its cross-sectional nature which does not allow the causal relationship between FOF, falls and gait to be determined although future waves will allow this to be teased out. In this study, FOF was assessed using a quick, simple and clinically useful dichotomous question similar to what has been used in other studies $[10,16,29]$. While self-efficacy scales are sometimes used $[9,10]$, they reflect confidence in the ability to carry out a range of activities, therefore they are not exactly the same construct and are not directly comparable [30]. Finally, gait variability for each participant was calculated from a minimum of six strides. While this may be sufficient for mean spatiotemporal gait parameters, a greater number of strides may be necessary for acceptable reliability in gait variability measures. This was not possible given the large scale nature of this study, although the approach used is comparable to existing research.

In conclusion, FOF is associated with a deterioration in gait characteristics in normal and dual task conditions independent of physical, mental and cognitive function and these gait changes are most pronounced in those who restrict activities as a result of FOF. These gait changes may increase the risk of falls in those with FOF, however changes especially increased step width may also reflect positive, compensatory adaptations to FOF. Longitudinal data is required to examine whether these potential stabilising gait changes actually reduce the risk of falls and if this differs between those with and without activity restriction. FOF did not affect the ability to complete cognitive tasks while walking which is important for everyday living. The results also highlight the importance of treating underlying health impairments and preventing the transition from FOF to activity restriction.

\section{Conflict of interest statement}

No conflicts of interest.

\section{References}

[1] Arfken CL, Lach HW, Birge SJ, Miller JP. The prevalence and correlates of fear of falling in elderly persons living in the community. American Journal of Public Health 1994;84(4):565-70. 
[2] Scheffer AC, Schuurmans MJ, van Dijk N, van der Hooft T, de Rooij SE. Fear of falling: measurement strategy, prevalence, risk factors and consequences among older persons. Age and Ageing 2008;37(1):19-24.

[3] Vellas BJ, Wayne SJ, Romero LJ, Baumgartner RN, Garry PJ. Fear of falling and restriction of mobility in elderly fallers. Age and Ageing 1997;26(3):189-93.

[4] Zijlstra GAR, van Haastregt JCM, van Eijk JTM, van Rossum E, Stalenhoef PA Kempen GIJM. Prevalence and correlates of fear of falling, and associated avoidance of activity in the general population of community-living older people. Age and Ageing 2007;36(3):304-9.

[5] Friedman SM, Munoz B, West SK, Rubin GS, Fried LP. Falls and fear of falling: which comes first? A longitudinal prediction model suggests strategies for primary and secondary prevention. Journal of the American Geriatrics Society 2002;50(8):1329-35.

[6] Delbaere K, Crombez G, Vanderstraeten G, Willems T, Cambier D. Fear-related avoidance of activities, falls and physical frailty. A prospective communitybased cohort study. Age and Ageing 2004;33(4):368-73.

[7] Martin FC, Hart D, Spector T, Doyle DV, Harari D. Fear of falling limiting activity in young-old women is associated with reduced functional mobility rather than psychological factors. Age and Ageing 2005;34(3):281-7.

[8] Brouwer B, Musselman K, Culham E. Physical function and health status among seniors with and without a fear of falling. Gerontology 2004;50(3):135-41.

[9] Chamberlin ME, Fulwider BD, Sanders SL, Medeiros JM. Does fear of falling influence spatial and temporal gait parameters in elderly persons beyond changes associated with normal aging? Journals of Gerontology Series A Biological Sciences and Medical Sciences 2005;60(9):1163-7.

[10] Reelick MF, van Iersel MB, Kessels RPC, Rikkert MGMO. The influence of fear of falling on gait and balance in older people. Age and Ageing 2009;38(4): 435-40.

[11] Kirkwood RN, de Souza Moreira B, Vallone MLDC, Mingoti SA, Dias RC, Sampaio $\mathrm{RF}$. Step length appears to be a strong discriminant gait parameter for elderly females highly concerned about falls: a cross-sectional observational study. Physiotherapy 2011;97(2):126-31.

[12] Menz HB, Lord SR, Fitzpatrick RC. Age-related differences in walking stability. Age and Ageing 2003;32(2):137-42.

[13] Lord SR, Lloyd DG, Li SK. Sensori-motor function, gait patterns and falls in community-dwelling women. Age and Ageing 1996;25(4):292-9.

[14] Lemke MR, Wendorff T, Mieth B, Buhl K, Linnemann M. Spatiotemporal gait patterns during over ground locomotion in major depression compared with healthy controls. Journal of Psychiatric Research 2000;34(4-5):277-83.

[15] Hausdorff JM, Edelberg HK, Mitchell SL, Goldberger AL, Wei JY. Increased gait unsteadiness in community-dwelling elderly fallers. Archives of Physical Medicine and Rehabilitation 1997;78(3):278-83.
[16] Rochat S, Büla CJ, Martin E, Seematter-Bagnoud L, Karmaniola A, Aminian K, et al. What is the relationship between fear of falling and gait in wellfunctioning older persons aged 65 to 70 years? Archives of Physical Medicine and Rehabilitation 2010;91(6):879-84.

[17] Gage WH, Sleik RJ, Polych MA, McKenzie NC, Brown LA. The allocation of attention during locomotion is altered by anxiety. Experimental Brain Research 2003;150(3):385-94.

[18] Gschwind YJ, Bridenbaugh SA, Kressig RW. Gait disorders and falls. GeroPsych The Journal of Gerontopsychology and Geriatric Psychiatry 2010;23(1):21-32.

[19] Kearney PM, Cronin H, O’Regan C, Kamiya Y, Savva GM, Whelan B, et al. Cohort profile: the Irish Longitudinal Study On Ageing. International Journal of Epidemiology 2011;40(4):877-84.

[20] D’Elia LF, Satz P, Uchiyama CL, White T. Color trails test. Professional manual. Odessa, FL: Psychological Assessment Resources; 1996.

[21] Bland JM, Altman DG. Statistics notes: measurement error. British Medical Journal 1996;313(7059):744.

[22] Verghese J, Holtzer R, Lipton RB, Wang C. Quantitative gait markers and incident fall risk in older adults. Journals of Gerontology Series A Biological Sciences and Medical Sciences 2009;64A(8):896-901.

[23] Curcio C-L, Gomez F, Reyes-Ortiz CA. Activity restriction related to fear of falling among older people in the colombian andes mountains. Journal of Aging and Health 2009;21(3):460-79.

[24] Murphy SL, Williams CS, Gill TM. Characteristics associated with fear of falling and activity restriction in community-living older persons. Journal of the American Geriatrics Society 2002;50(3):516-20.

[25] Menz HB, Lord SR, Fitzpatrick RC. A structural equation model relating impaired sensorimotor function, fear of falling and gait patterns in older people. Gait and Posture 2007;25(2):243-9.

[26] Latt M, Menz H, Fung V, Lord S. Walking speed, cadence and step length are selected to optimize the stability of head and pelvis accelerations. Experimental Brain Research 2008;184(2):201-9.

[27] Menz HB, Lord SR, Fitzpatrick RC. Acceleration patterns of the head and pelvis when walking are associated with risk of falling in community-dwelling older people. Journals of Gerontology Series A Biological Sciences and Medical Sciences 2003;58(5):M446-52.

[28] Callisaya ML, Blizzard L, Schmidt MD, Martin KL, McGinley JL, Sanders LM, et al. Gait, gait variability and the risk of multiple incident falls in older people: a population-based study. Age and Ageing 2011;40(4):481-7.

[29] Brown LA, Doan JB, McKenzie NC, Cooper SA. Anxiety-mediated gait adaptations reduce errors of obstacle negotiation among younger and older adults: implications for fall risk. Gait and Posture 2006;24(4):418-23.

[30] Legters K. Fear of falling. Physical Therapy 2002;82(3):264-72. 